\title{
KÄHLER MANIFOLDS WITH ALMOST NON-NEGATIVE BISECTIONAL CURVATURE*
}

\author{
FUQUAN FANG ${ }^{\dagger}$
}

\begin{abstract}
The main purpose of this paper is to study the topology of Kähler manifolds with almost non-negative bisectional curvature. Among others we prove that for simply connected $n$ dimensional Kähler manifolds $M$ of sectional curvature $K \leq \Lambda$, there exists a universal positive constant $\varepsilon(n, \Lambda)$, depending only on the dimension $n$ and $\Lambda$, such that if the bisectional curvature $H$ and the diameter of $M$ satisfy, $H \cdot \operatorname{diam}^{2}(M) \geq-\varepsilon(n, \Lambda)$, then $M$ is diffeomorphic to the product $M_{1} \times \cdots \times M_{k}$, where each $M_{i}$ is a simply connected $C^{1, \alpha}$-Kähler manifold with second Betti number $b_{2}\left(M_{i}\right)=1$ for any prescribed real number $\alpha \in(0,1)$. Furthermore, if $M$ is Kähler-Einstein, then $M_{i}$ are all biholomorphic to irreducible Kähler Hermitian symmetric spaces. In the non-simply connected case, we prove that $M$ is a holomorphic fiber bundle over the Jacobian $\mathcal{J}(M)$.
\end{abstract}

0. Introduction. Let $M$ be a compact complex manifold. We say $M$ has almost nonnegative bisectional curvature, if for any positive constant $\varepsilon$, there is a Hermitian metric $g$ on $M$ whose bisectional curvature $H$ satisfies that $H \cdot \operatorname{diam}\left(M_{g}\right)^{2} \geq-\varepsilon$. Besides Hermitian manifolds of non-negative bisectional curvature, there are many examples of complex manifolds of almost non-negative bisectional curvature but do not admit any Hermitian metric of non-negative bisectional curvature (c.f. Section 1.)

When the Hermitian manifold is Kählerian, the uniformization theorem of Mok [Mo] (generalized Frankel conjecture, compare Siu-Yau [SY]) asserts that a simply connected compact Kähler manifold $M$ with non-negative bisectional curvature is biholomorphic to the product of

$$
P(\mathbb{C})^{m_{1}} \times \cdots \times P(\mathbb{C})^{m_{k}} \times N_{1} \cdots \times N_{l}
$$

where $N_{i}, 1 \leq i \leq l$, are irreducible Kähler Hermitian symmetric spaces of rank at least 2. The Mok theorem depends on an earlier decomposition theorem of Howard-SmythWu [HSW], Mori's celebrated work [Mo] and Hamilton's heat equation technique.

A natural question is whether one can extend the Mok theorem and the HowardSmyth-Wu theorem to Kähler manifold of almost non-negative bisectional curvature. In this paper we will prove, among others, for simply connected $n$-dimensional Kähler manifold $M$ with sectional curvature $K \leq \Lambda$, there exists a universal positive constant $\varepsilon(n, \Lambda)$, depending only on the dimension $n$ and $\Lambda$, such that if the bisectional curvature $H$ and the diameter of $M$ satisfy, $H \cdot \operatorname{diam}^{2}(M) \geq-\varepsilon(n, \Lambda)$, then $M$ is diffeomorphic to the product $M_{1} \times \cdots \times M_{k}$, where each $M_{i}$ is a simply connected $C^{1, \alpha}$-Kähler manifold with second Betti number $b_{2}\left(M_{i}\right)=1$ for any prescribed real number $\alpha \in(0,1)$. Furthermore, if $M$ is Kähler-Einstein, then $M_{i}$ are all biholomorphic to irreducible Kähler Hermitian symmetric spaces. In the non-simply connected case, we prove that $M$ is a holomorphic fiber bundle over the Jacobian $\mathcal{J}(M)$.

Now we start to state our main results.

For convenience, let $\mathcal{M}(n, \Lambda)$ denote the set of all $n$-dimensional Kähler manifolds so that $K \leq \Lambda$.

\footnotetext{
* Received November 15, 2001; accepted for publication April 23, 2002.

† Nankai Institute of Mathematics, Nankai University, Tianjin 300071, P.R.C. (ffang@nankai.edu.cn). Instituto de Matematica, Universidade Federal Fluminense, Niteroi 24005, RJ, Brasil (fuquan@mat.uff.br). Supported by NSFC Grant 19741002, RFDP, CNPq and the Qiu-Shi Foundation.
} 
THEOREM A. Let $M \in \mathcal{M}(n, \Lambda)$ be a simply connected compact Kähler manifold. Then there exists a constant $\varepsilon(n, \Lambda)$, depending only on $n$ and $\Lambda$, such that if $H$. $\operatorname{diam}(M)^{2} \geq-\varepsilon(n, \Lambda)$, then $M$ is diffeomorphic to the product $M_{1} \times \cdots \times M_{k}$, where $M_{i}, 1 \leq i \leq k$, are simply connected $C^{1, \alpha}$-Kähler manifolds with second Betti number $b_{2}\left(M_{i}\right)=1$ for any prescribed real number $\alpha \in(0,1)$.

If $M \in \mathcal{M}(n, \Lambda)$ is Kähler-Einstein, we get an improved splitting theorem

THEOREM B. Let $M \in \mathcal{M}(n, \Lambda)$ be a simply connected Kähler-Einstein manifold. Then there exists a constant $\varepsilon(n, \Lambda)$, depending only on $n$ and $\Lambda$, such that if $H$. $\operatorname{diam}^{2}(M) \geq-\varepsilon(n, \Lambda)$, then $M$ is diffeomorphic to the product

$$
P(\mathbb{C})^{m_{1}} \times \cdots \times P(\mathbb{C})^{m_{k}} \times N_{1} \cdots \times N_{l}
$$

where $N_{i}, 1 \leq i \leq l$, are irreducible Kähler-Einstein Hermitian symmetric spaces of rank at least 2 .

Observe that for $M$ in Theorem A with $\varepsilon$ sufficiently small, the Ricci curvature of $M$ is almost non-negative. By [FY] and [CC] we know that the fundamental group $\pi_{1}(M)$ is virtually nilpotent. In other words, $M$ has a finite cover whose fundamental group is nilpotent. Note that for a finitely generated infinite nilpotent group, its first Betti number is nonzero. The following is a complex analogue of [Ya], which proves $M$ is a bundle over a torus $T^{b}$.

TheOREM C. Let $M \in \mathcal{M}(n, \Lambda)$. Then there is a positive constant $\varepsilon(n, \Lambda)$ such that if $H \cdot \operatorname{diam}^{2}(M) \geq-\varepsilon$, then there is a holomorphic fibration $\pi: M \rightarrow \mathcal{J}(M)$, where $\mathcal{J}(M)$ is the Jacobian of $M$, a complex torus of dimension $\frac{1}{2} b_{1}$.

Our next result gives an estimate for the Hodge number $h^{1,1}$, which may be viewed as a complex analogue of the Bochner-Gallot-Gromov first Betti number estimate (cf. $[\mathrm{Ga}])$.

Theorem D. Let $M \in \mathcal{M}(n, \Lambda)$. Then there is a positive constant $\varepsilon(n, \Lambda)$ such that if $H \cdot \operatorname{diam}^{2}(M) \geq-\varepsilon(n, \Lambda)$, then $h^{1,1}(M) \leq n$.

Now let us start to indicate the idea for the proof of the Theorem A.

Suppose not. Then there is a sequence of simply connected Kähler manifolds $M_{i} \in \mathcal{M}(n, \Lambda)$ such that the bisectional curvature $H_{i} \geq-(0.1)^{i}$. By the Gromov precompactness theorem, $\left\{M_{i}\right\}$ has a Gromov-Hausdorff limit $X$. By $[\mathrm{FR}]$ we know that $X$ is a manifold of the same dimension $n$, and moreover, for every sufficiently large $i$ (say $i \geq N$ ), there is a diffeomorphism $f_{i}: M_{i} \rightarrow X$. Moreover, the almost complex structure $\tilde{J}_{i}=\left(d f_{i}\right) J_{i}\left(d f_{i}\right)^{-1}$ converges to an almost complex structure $J_{\infty}$ on $T X$ which is integrable in $C^{1, \alpha}$-class, for any prescribed real number $\alpha \in(0,1)$. By the Newslander-Nirenberg theorem for $C^{1, \alpha}$-class (cf. Theorem 1.5) $\left(X, J_{\infty}\right)$ is a complex manifold. The pullback metrics $\left(\left(f_{i}\right)^{-1}\right)^{*} g_{i}$ converge to a $C^{1, \alpha}$ metric $g_{\infty}$ on $X$, by the well-known Cheeger-Gromov theorem. This metric is indeed a Kähler metric on $X$, compatible with the complex structure $J_{\infty}$.

If $h^{1,1}\left(M_{i}\right)=k$, we will prove there are $k$ linearly independent harmonic $(1,1)$ forms in $X$ of $C^{1, \alpha}$ class which are all parallel. Using these parallel forms we obtain a parallel decomposition of the tangent bundle $T X$ into $k$ distributions. This implies that the holonomy group of $X$ splits into the product of $k$ factors. Now by the de Rham decomposition theorem in $C^{1, \alpha}$-class (cf. Theorem 1.4) we conclude that $X=X_{1} \times \cdots \times X_{k}$, where each factor $X_{i}$ is a simply connected $C^{1, \alpha}$-Kähler manifold. 
On the other hand, we will prove a vanishing theorem for holomorphic $p$-forms (for any $p>0$ ) in simply connected Kähler manifolds with almost non-negative bisectional curvature. Therefore by the Hodge duality theorem $h^{2,0}\left(M_{i}\right)=h^{0,2}\left(M_{i}\right)=0$ and $b_{2}\left(M_{i}\right)=h^{2,0}\left(M_{i}\right)+h^{1,1}\left(M_{i}\right)+h^{0,2}\left(M_{i}\right)=k$. This implies that $b_{2}(X)=k$ and so $b_{2}\left(X_{1}\right)=\cdots=b_{2}\left(X_{k}\right)=1$, since each factor is a compact Kähler manifold. A contradiction. This together implies Theorem A.

The difficulty to prove $X_{i}$ is a Kähler Hermitian symmetric space occurs since the limit metric on $X$ is not necessarily smooth. However, if $M_{i}$ is Einstein, by the Einstein equation one can improve the regularity of the convergence. In particular, we get a smooth Kähler metric on $X$ with non-negative bisectional curvature. Therefore the Mok Theorem applies to show that each $X_{i}$ is an irreducible Kähler Hermitian symmetric space. Therefore Theorem B follows.

Theorem $\mathrm{C}$ follows along a similar strategy of [Ya], by using holomorphic forms instead of harmonic forms.

For the proof of Theorem D we show that there are $h^{1,1}$ harmonic $(1,1)$-forms on $M$ which are linearly independent at every point. In particular, this implies a decomposition of the tangent bundle $T M$ into $h^{1,1}$ complex sub-bundles. For the dimension reasoning we know that $h^{1,1} \leq n$.

We should like to remark that our approach in this paper does not imply an estimate for the constant $\varepsilon(n, \Lambda)$. A search for the precise bound will be extremely worthwhile.

In concluding this section we conjecture the following

CONJECTURE E. There is a positive constant $\varepsilon(n)$ depending only on the dimension, such that if $M$ is a simply connected compact Kähler manifold whose holomorphic bisectional curvature satisfies

$$
H \cdot \operatorname{diam}(M)^{2} \geq-\varepsilon(n)
$$

and the second Betti number $b_{2}(M)=1$, then $M$ is either diffeomorphic to a complex projective space or an irreducible Kähler-Hermitian symmetric space of rank $\geq 2$.

Acknowledgement: The author would like to thank Manfredo do Carmo for helpful discussions, Michael Anderson and H. Wu for their comments. Special thanks go to the referee for some very constructive suggestions.

1. Preliminaries. In this section we give some necessary preliminary results needed in next sections.

a). Example (manifolds of almost non-negative bisectional curvature)

EXAMPLE 1.1. Let $N$ be the complex Heisenberg group and $\Gamma$ the Gaussian integer lattice

$$
N=\left\{\left(\begin{array}{lll}
1 & x & z \\
0 & 1 & y \\
0 & 0 & 1
\end{array}\right) ; x, y, z \in \mathbb{C}\right\}, \quad \Gamma=\left\{\left(\begin{array}{lll}
1 & c & a \\
0 & 1 & b \\
0 & 0 & 1
\end{array}\right) ; a, b, c \in \mathbb{Z} \oplus \mathbb{Z} \sqrt{-1}\right\}
$$

The quotient space $M=N / \Gamma$ is a complex analytic variety, and $h^{1,0}(M)=h^{0,1}(M)=$ 
2. For each $\varepsilon>0$, we may define a right invariant Hermitian metric $h_{\varepsilon}$ on $M$ by

$$
\left(\begin{array}{ccc}
0 & w & u \\
0 & 0 & v \\
0 & 0 & 0
\end{array}\right) \in \mathcal{N} ; \quad\left\|\left(\begin{array}{ccc}
0 & w & u \\
0 & 0 & v \\
0 & 0 & 0
\end{array}\right)\right\|=\varepsilon^{2}|u|^{2}+|v|^{2}+|w|^{2}
$$

where $\mathcal{N}$ is the Lie algebra of $N$. Note that the sectional curvature and diameter satisfy that $\left|K\left(h_{\varepsilon}\right)\right| \leq 24 \varepsilon^{2}$, diam $\left(M, g_{\varepsilon}\right) \leq 2$. In particular, $M$ has almost nonnegative bisectional curvature (compare $[Y a]$ ).

The above manifold $M=N / \Gamma$ is a complex nil-manifold, whose fundamental group is not virtually abelian. By the Cheeger-Gromoll theorem [CG] we know that $M$ does not admit any metric of non-negative Ricci curvature. On the other hand, by a theorem of Benson-Gordon-Hasegawa [BG][Ha] no nil-manifold other than tori admits a Kähler structure. More generally, it is proved that no fiber bundle over a non-torus nil-manifold with fiber $P(\mathbb{C})^{m}$ admits a Kähler structure. It seems natural to ask the following problem:

Problem 1.2. Let $M$ be a Kähler manifold of almost non-negative bisectional curvature. Is its fundamental group virtually abelian?

\section{b). Gromov-Hausdorff convergence}

For subsets $A$ and $B$ of a metric space $X$, recall that the Hausdorff distance $d_{H}(A, B)$ is the infimum of radii $\varepsilon$ so that the $\varepsilon$ neighborhood of $A$ (resp. $B$ ) include $B$ (resp. $A$.) For two abstract metric spaces $A$ and $B$, a metric on the disjoint union $A \amalg B$ is called admissible if it restricts to the metrics on $A$ and $B$ respectively. The Gromov-Hausdorff distance $d_{G H}(A, B)$ is the infimum of Hausdorff distances of $A$ and $B$ as subsets of $A \coprod B$, with respect to all possible admissible metrics.

The following compactness theorem is important for our applications (cf. [Ch][GLP]).

THEOREM 1.3 [CHEEGER-GROMOV]. Let $M_{i}$ be a sequence of compact Riemannian manifolds whose sectional curvature, diameter, and injectivity radius satisfy

$$
\lambda \leq K \leq \Lambda, \quad \text { diam } \leq d, \quad i_{M}>i_{0},
$$

where the constants are independent of $i$. Then, replacing $M_{i}$ by a subsequence if necessary, $M_{i}$ converges to a metric space $X$, such that

(i) $X$ is a differentiable manifold;

(ii) there is a diffeomorphsim $f_{i}: X \rightarrow M_{i}$ for all sufficiently large $i$;

(iii) the pullback metrics $f_{i}^{*}\left(g_{i}\right)$ converges in $C^{1, \alpha}$-class to a $C^{1, \alpha}$ (resp. $\left.L^{2, p}\right)$ Riemannian metric $g_{\infty}$ in $X$, for any prescribed real number $\alpha \in(0,1)$ (resp. positive integer $p>1$ ).

For the sake of simplicity, in the rest of the paper we fix the real number $\alpha \in(0,1)$ (resp. the interger $p>n$ ).

The classical de Rham decomposition theorem holds for Kähler manifolds in $C^{1, \alpha}$ class with identical proof.

THEOREM 1.4 [LIC]. If $M$ is a simply connected complete $C^{1, \alpha}$-Kähler manifold whose holonomy representation splits into a direct sum $A_{1} \times A_{2} \times \cdots \times A_{k} \subset U\left(n_{1}\right) \times$ $U\left(n_{2}\right) \times \cdots \times U\left(n_{k}\right) \subset U\left(n_{1}+\cdots+n_{k}\right)$, then $M$ is isometric to a product $M_{1} \times \cdots \times M_{k}$ of $C^{1, \alpha}$-Kähler manifolds $M_{1}, \cdots, M_{k}$ of dimensions $n_{1}, \cdots, n_{k}$. 
If every $M_{i}$ in Theorem 1.3 is a complex Hermitian manifold, then the complex structure $\tilde{J}_{i}=\left(d f_{i}\right)^{-1} J_{i}\left(d f_{i}\right)$ on the tangent bundle $T X$ converges to an almost complex structure $J_{\infty}$ in $X$, which is integrable in $C^{1, \alpha}$-class. The following version of the well-known Newlander-Nirenberg theorem implies that $J_{\infty}$ is a complex structure.

THEOREM $1.5[\mathrm{NW}]$. Let $(X, J)$ be an almost complex manifold with an integrable almost complex structure $J$ in $C^{1, \alpha}$-class. Then $(X, J)$ is a complex manifold.

Therefore, the limit $X$ is also a complex manifold with a $C^{1, \alpha}$ Hermitian metric $g_{\infty}$. In general, the metric $g_{\infty}$ is not $C^{2}$. However, if $M_{i}$ are all Einstein, then from the ellipticity regularity of the Einstein equation one can prove the limit metric $g_{\infty}$ is smooth, by the work of M. Anderson [An].

THEOREM 1.6 [ANDERSON]. Let $M_{i}$ be a sequence of compact Einstein manifolds whose sectional curvature, diameter, and injectivity radius satisfy

$$
\lambda \leq K \leq \Lambda, \quad \text { diam } \leq d, \quad i_{M}>i_{0},
$$

where the constants are independent of $i$. Then $M_{i}$ has a subsequence converging to a $C^{\infty}$ Riemannian manifold $X$, such that

(i) there is a diffeomorphsim $f_{i}: X \rightarrow M_{i}$ for all sufficiently large $i$;

(ii) the pullback metrics $f_{i}^{*}\left(g_{i}\right)$ converges in $C^{\infty}$-class to a Riemannian metric $g_{\infty}$.

Let us continue to use the same notations in the Introduction. Observe that for any flat manifold $M$ we have $H \cdot \operatorname{diam}^{2}(M)=0 \geq-\varepsilon$. Obviously the diameter can be made arbitrarily large or small by scaling and so is for the injectivity radius. This also happens for simply connected manifold. For example, the Berger spheres provide an example of simply connected positively curved manifold of bounded curvature whose injectivity radius can be arbitrarily small. However, for Kähler manifolds we have a uniform positive lower bound for the injectivity radii by the following theorem in $[F R]$.

TheOREM 1.7. Let $M \in \mathcal{M}(n, \Lambda)$. If $M$ has finite fundamental group, and $H$. $\operatorname{diam}^{2}(M) \geq-\varepsilon$, then the injectivity radius $i_{M}$ has a positive lower bound, depending only on $n, \Lambda$ and $\varepsilon$.

\section{Convergence of harmonic forms.}

\section{a). Harmonic coordinates}

A key step in this paper is to establish a convergence theorem for harmonic $(1,1)$ forms in Kähler manifolds. To start with, let us recall some necessary preliminary on harmonic coordinates.

By definition, a local coordinate $\left(h^{1}, \ldots, h^{n}\right)$ is harmonic if each component is a harmonic function, i.e., $\triangle h^{i}=0$ for $i=1, \ldots, n$, where $\triangle$ is the Laplacian opertator. In a harmonic coordinate, the Ricci curvature of the metric tensor $g$ satisfies the equation

$$
\left(\mathrm{Ric}_{g}\right)_{i j}=-\frac{1}{2} \triangle g_{i j}+Q(g, \partial g)
$$

here $Q(.,$.$) is a quadratic form of its variables (c.f. [Pe]).$

Theorem 2.1 [Anderson, Jost, Karcher-Jost]. Given $\epsilon_{0}>0$ and $\alpha \epsilon$ $(0,1)$. Assume the Riemannian manifold $(M, g)$ satisfies the conditions that the injectivity radius $i_{g} \geq \epsilon_{0}$ and the sectional curvature $\left|K_{g}\right| \leq 1$. Then there exist $r>0$ 
depending only on $\epsilon_{0}, n$ and a constant $C$ depending only on $\epsilon_{0}, n, \alpha$ (resp. $\left.\epsilon_{0}, n, p\right)$ such that there is a harmonic coordinate system $\left\{h^{i}, i=1, \cdots, n\right\}$ on $B(x, r)$ satisfying

$$
\left.\left\|g_{i j}\right\|_{C^{1, \alpha}} \leq C \text { (resp. }\left\|g_{i j}\right\|_{L^{2, p}} \leq C\right)
$$

where $g_{i j}=g\left(\frac{\partial}{\partial h^{i}}, \frac{\partial}{\partial h^{j}}\right)$ and $L^{2, p}$ is the Sobolev norm.

For a Kähler manifold, the holomorphic coordinate is the most natural harmonic coordinate.

\section{b). Convergence of harmonic real $(1,1)$-forms}

Let $M$ be a Kähler manifold. Let $\left\{V_{i}\right\}$ be a local frame field of type $(1,0)$ and let $\left\{\omega^{i}\right\}$ be its dual coframe field. Let $\Delta=\bar{\partial} \bar{\partial}^{*}+\bar{\partial}^{*} \bar{\partial}$ be the complex Laplacian operator on $(p, q)$-forms. On a Kähler manifold, $\Delta=\partial \partial^{*}+\partial^{*} \partial$.

Let $D_{X Y}^{2}=D_{X} D_{Y}-D_{D_{X} Y}$ denote the second order covariant differential, $R_{X Y}=$ $-D_{X} D_{Y}+D_{Y} D_{X}+D_{[X, Y]}$ denote the curvature tensor acting on forms of all degrees as a derivation and $i(X)$ denotes the interior product.

The well-known Weitzenböck formula for Hodge-Laplacian operator on Kähler manifold (cf. [Wu2]) reads

WEITZENBÖCK FORMUlA.

$$
\Delta=-\sum_{i} D_{V_{i} \bar{V}_{i}}^{2}-\sum_{i, j} \bar{\omega}^{i} \wedge i\left(\bar{V}_{j}\right) R_{V_{j} \bar{V}_{i}}
$$

LEMMA 2.2. Let $M_{i} \in \mathcal{M}(n, \Lambda)$. Let $\xi_{i} \in H^{1,1}\left(M_{i}\right)$ be a sequence of harmonic $(1,1)$-forms with $L^{2}$-norm $\left\|\xi_{i}\right\|_{0,2}=1$. If the injectivity radii $i_{M_{i}} \geq i_{0}$, a uniform constant, then there exists a positive constant $\varepsilon(n, \Lambda)$ so that if $H_{i} \cdot \operatorname{diam}^{2}\left(M_{i}\right) \geq$ $-\varepsilon(n, \Lambda)$, then the pullback forms $f_{i}^{*}\left(\xi_{i}\right)$ contains a converging subsequence in $C^{1, \alpha}$ class (for any $\alpha<1$ ) whose limit is a non-trivial parallel $(1,1)$-form $\hat{\xi}$ with respect to the limit metric $g_{\infty}$, where $f_{i}$ are as in Theorem 1.3.

Proof. Observe that the sectional curvature of $M_{i}$ are uniformly bounded from below. By Theorem 2.1 we can assume $B_{i}^{1}(r), \cdots, B_{i}^{m}(r)$ be a cover of harmonic coordinates of $M_{i}$ with radii $r$, independent of $i$. For all $i>N$ large and all $q$, we get diffeomorphisms $f_{i}^{q}: B^{q}=B_{N}^{q}(r) \rightarrow B_{i}^{q}(r)$. The pullback metric tensors $\left(f_{i}^{q}\right)^{*}\left(g_{i}\right)$ converges to a $C^{1, \alpha}\left(\right.$ resp. $\left.L^{2, p}\right)$ metric $g_{\infty}$ on $B^{q}$. The $(1,1)$-forms $\eta_{l}=\left(f_{l}^{q}\right)^{*}\left(\left.\xi_{l}\right|_{B_{l}^{q}(r)}\right)$ on $B^{q}$, satisfy the equation

$$
\sum_{i} D_{V_{i} \bar{V}_{i}}^{2} \eta_{l}+\sum_{i, j} \bar{\omega}^{i} \wedge i\left(\bar{V}_{j}\right) R_{V_{j} \bar{V}_{i}}\left(\eta_{l}\right)=0
$$

since $\Delta\left(\eta_{l}\right)=0$, by the above Weitzenböck formula.

This is an elliptic system of order 2 . It is easy to check that

(i) the second order terms coefficients are uniformly $C^{0}$-bounded (independent of the indices $l$ );

(ii) the first order terms coefficients are uniformly $C^{0, \alpha}$-bounded;

(iii) the zero order terms coefficients are uniformly $L^{p}$-bounded, by Theorem 2.1 .

By the standard elliptic estimate (c.f. $[\mathrm{ADN}]$ ) we conclude that the Sobolev $L^{2, p_{-}}$ norms of $\eta_{l}$ are uniformly bounded, for any $p<\infty$. Therefore, $\eta_{l}$ has a subsequence converging in $L^{2, p^{\prime}}$ for any $p^{\prime}<p$. Note that $L^{2, p} \subset C^{1,1-\frac{n}{p}}$. Therefore there is a $C^{1, \alpha}$-convergence subsequence $\eta_{l} \rightarrow \eta$. 
To prove $\hat{\xi}$ is parallel with respect to the metric $g_{\infty}$, it suffices to show that $\eta$ is parallel with respect to the $C^{1, \alpha}$-limit metric of $\left(f_{i}^{q}\right)^{*} g_{i}$ on $B^{q}$.

Note that for a real $(1,1)$-form, the Weitzenböck formula implies that (c.f. [Wu2])

$$
-\Delta\left|\eta_{l}\right|^{2}=\sum_{i}\left(\left|D_{\bar{V}_{i}} \eta_{l}\right|^{2}+\left|D_{V_{i}} \eta_{l}\right|^{2}\right)-2\left\langle\sum_{i, j} \bar{\omega}^{i} \wedge i\left(\bar{V}_{j}\right) R_{V_{i} \bar{V}_{j}} \eta_{l}, \eta_{l}\right\rangle
$$

Let

$$
F\left(\eta_{l}\right)=\left\langle\sum_{i, j} \bar{\omega}^{i} \wedge i\left(\bar{V}_{j}\right) R_{V_{i} \bar{V}_{j}} \eta_{l}, \eta_{l}\right\rangle
$$

We may write $\eta_{l}$ locally as

$$
\eta_{l}=\sum_{i} \sqrt{-} 1 \eta_{l}^{i} \omega^{i} \wedge \bar{\omega}^{i}
$$

By [Wu2] one has the following formula

$$
F\left(\eta_{l}\right)=\frac{1}{2} \sum_{j, k} R_{j j k k}^{l}\left(\eta_{l}^{j}-\eta_{l}^{k}\right)^{2}
$$

where $-R_{j j k k}^{l}$ is the bisectional curvature of $M_{l}$ at the complex plane spanned by $V_{j}, V_{k}$. Therefore we get

$$
-\Delta\left|\eta_{l}\right|^{2}=\sum_{i}\left|D_{\bar{V}_{i}} \eta_{l}\right|^{2}+\sum_{i}\left|D_{V_{i}} \eta_{l}\right|^{2}-\sum_{j, k} R_{j j k k}^{l}\left(\eta_{l}^{j}-\eta_{l}^{k}\right)^{2}
$$

By integrating both sides and taking limit we get

$$
\lim _{l}\left(\sum_{i}\left|D_{\bar{V}_{i}} \eta_{l}\right|^{2}+\sum_{i}\left|D_{V_{i}} \eta_{l}\right|^{2}\right)=0
$$

since the integration of $-F\left(\eta_{l}\right)$ has limit non-negative, by the assumption of almost non-negativity of the bisectional curvature. Therefore $\lim _{l} D_{V_{i}} \eta_{l}=0$ and $\lim _{l} D_{\bar{V}_{i}} \eta_{l}=$ 0 . This implies that $D_{V_{i}}^{\infty} \eta=D_{\bar{V}_{i}}^{\infty} \eta=0$, where $D^{\infty}$ is the covariant derivative of the the limit metric $g_{\infty}$. This proves that $\eta$ is parallel in the metric $g_{\infty}$, so $\hat{\xi}$ is also parallel.

Obviously, $\|\hat{\xi}\|_{0,2}=\lim \left\|\xi_{i}\right\|_{0,2}=1$. In particular, $\hat{\xi}$ is nontrivial. The desired result follows.

As a direct corollary we have

Proposition 2.3. Let $M_{i} \in \mathcal{M}(n, \Lambda)$. Suppose that $h^{1,1}\left(M_{i}\right)=k$ for all $i$. Let $\left\{\xi_{i}^{1}, \cdots, \xi_{i}^{k}\right\}$ be a sequence of orthonormal basis for $\left(H^{1,1}\left(M_{i}\right),\|\cdot\|_{0,2}\right)$. If the injectivity radii $i_{M_{i}} \geq i_{0}$, a uniform constant, then there exists a positive constant $\varepsilon(n, \Lambda)$ so that if $H_{i} \cdot \operatorname{diam}^{2}\left(M_{i}\right) \geq-\varepsilon(n, \Lambda)$, then, passing to a subsequence if necessary, $\left\{f_{i}^{*}\left(\xi_{i}^{1}\right), \cdots, f_{i}^{*}\left(\xi_{i}^{k}\right)\right\}$ converges in $C^{1, \alpha}$-class to orthonormal parallel $(1,1)$-forms $\left\{\hat{\xi}^{1}, \cdots, \hat{\xi}^{k}\right\}$ with respect to the limit metric $g_{\infty}$.

Next we consider a sequence of manifolds whose injectivity radii tend to zero.

Let $M_{i} \in \mathcal{M}(n, \Lambda)$. Let $\xi_{i} \in \Lambda^{1,1}\left(M_{i}\right)$ be real harmonic $(1,1)$-forms with normalized $L^{2}$-norm $\left\|\xi_{i}\right\|_{0,2} / \sqrt{\operatorname{vol}\left(M_{i}\right)}=1$, where $\operatorname{vol}\left(M_{i}\right)$ is the volume of $M_{i}$. If $H_{i} \cdot \operatorname{diam}^{2}\left(M_{i}\right) \geq-1$, then the sectional curvature of $M_{i}$ are uniformly bounded from both sides. Therefore the conjugate radii have a uniform lower bound, say $2 r$. Let $B_{i}^{1}(2 r), \cdots, B_{i}^{m}(2 r)$ be the radii $2 r$ balls in the tangent spaces $T_{p_{k}} M_{i}, 1 \leq k \leq m$, 
so that the exponential maps $\exp _{p_{k}}: B_{i}^{k}(2 r) \subset T_{p_{k}} M_{i} \rightarrow M_{i}$ are immersions and $\exp _{p_{1}}\left(B_{i}^{1}(r)\right), \cdots, \exp _{p_{m}}\left(B_{i}^{m}(r)\right)$ is a cover of $M_{i}$. For every fixed $k$, consider the pullback metrics $\exp _{p_{k}}^{*}\left(g_{i}\right)$ in $B_{i}^{k}(2 r)$. Note that the injectivity radius at every point of $B_{i}^{k}(r)$ with the metric $\exp _{p_{k}}^{*}\left(g_{i}\right)$ have a uniform lower bound, independent of the index $i$. By Theorem 1.3 there is an integer $N$ such that for all $i>N$, there are diffeomorphisms $f_{i}^{k}: B^{k}:=B_{N}^{k}(r) \rightarrow B_{i}^{k}(r)$ so that the pullback metric tensors $\left(f_{i}^{k}\right)^{*}\left(\exp _{p_{k}}^{*}\left(g_{i}\right)\right)$ converge in $C^{1, \alpha}\left(\right.$ resp. $\left.L^{2, p}\right)$ class. Let $\eta_{i}=\left(f_{i}^{k}\right)^{*}\left(\exp _{p_{k}}^{*}\left(\xi_{i}\right)\right)$ be the pulled back $(1,1)$-forms in $B^{k}$.

Lemma 2.4. Let $M_{i} \in \mathcal{M}(n, \Lambda)$ and let $\eta_{i}$ be as above. There exists a positive constant $\varepsilon(n, \Lambda)$ so that if $H_{i} \cdot \operatorname{diam}^{2}\left(M_{i}\right) \geq-\varepsilon(n, \Lambda)$, then $\eta_{i}$ contains a $C^{1, \alpha}$ converging subsequence whose limit is a parallel $(1,1)$-form with respect to the limit metric in $B^{k}, 1 \leq k \leq m$.

Proof. Let $\xi_{i} \in \Lambda^{1,1}\left(M_{i}\right)$ be the real harmonic $(1,1)$-forms as above. By Peter Li [Li] Lemma 8 the pointwise $C^{0}$-norms $\left|\xi_{i}\right|$ satisfy the inequalities

$$
\Delta\left|\xi_{i}\right| \leq C\left|\xi_{i}\right|
$$

where $C$ is a constant depending only on the bound $\Lambda, \varepsilon(n, \Lambda)$ and $n$. This together with Lemma 20 in [Ga] implies that

$$
\left|\xi_{i}\right| \leq C\left\|\xi_{i}\right\|_{0,2} / \sqrt{\operatorname{vol}\left(M_{i}\right)}=C
$$

Therefore the $C^{0}$-norms $\left|\eta_{i}\right| \leq C$ for all $i$.

By (2.2.1) and the standard elliptic estimate [ADN] it follows that

$$
\left\|\eta_{i}\right\|_{2, p} \leq C_{2, p}
$$

for any $p<\infty$. As in the proof of Lemma 2.2, this implies that $\eta_{i}$ contains a convergence subsequence in $C^{1, \alpha}$ class.

By the technique of Yamaguchi (cf. [Ya] the proof of Prop. 2.2) and the formula $(2.2 .2)$ it follows that $\eta_{i}$ converges to a parallel $(1,1)$-form in $C^{1, \alpha}$-class. The desired result follows.

\section{c). A vanishing theorem for holomorphic $p$-forms}

It is well-known that a compact Kähler $m$-manifold with positive Ricci form has no non-trivial holomorphic $p$-forms, $p=1, \cdots, m$ (cf. [Be] page 323). The same result holds for simply connected compact Kähler manifold with non-negative bisectional curvature. The following vanishing result is a generalization of this fact to simply connected compact Kähler manifold with almost non-negative bisectional curvature.

TheOREM 2.5. Let $M \in \mathcal{M}(n, \Lambda)$ be a Kähler manifold. Suppose the fundamental group of $M$ is finite. Then there is a positive constant $\varepsilon(n, \Lambda)$ such that if $H \cdot \operatorname{diam}^{2}(M) \geq-\varepsilon(n, \Lambda)$, then $M$ does not have nonzero holomorphic $p$-forms for $p>0$, i.e. $h^{p, 0}(M)=0$.

In the above theorem the finiteness of the fundamental group of $M$ is necessary, since flat Kähler manifold fits in the class of the manifolds but with $h^{p, 0}(M)$ nonzero. The idea in proving Theorem 2.5 is by argument by contradiction, to show otherwise the fundamental group can never be finite. 
For a compact Kähler manifold $M \in \mathcal{M}(n, \Lambda)$ with metric $g$, a real harmonic $(1,1)$-form $\xi \in H^{1,1}(M)$ induces a linear self-adjoint transformation

$$
S: T M \rightarrow T M
$$

by setting $\xi(v, w)=g(S(J v), w)$ for all $v, w \in T M$, where $J$ is the complex structure. Let $\alpha_{1}(x), \cdots, \alpha_{n}(x)$ denote the eigenvalues of $\left.S\right|_{x}$, the restriction of $S$ on the tangent space $T_{x} M$. Obviously, if the $(1,1)$-form $\xi$ is parallel, then $\alpha_{1}(x), \cdots, \alpha_{n}(x)$ are constants. Moreover, if $\xi$ is not a multiple of $\omega$, these constants can not be all the same.

Lemma 2.7. Let $M_{i} \in \mathcal{M}(n, \Lambda)$ and let $\left\{\xi_{i}^{1}, \cdots, \xi_{i}^{k}\right\}$ be as in Proposition 2.3. Let $\left(X, J_{\infty}, g_{\infty}\right)$ be the Gromov-Hausdorff limit of the sequence $\left(M_{i}, J_{i}, g_{i}\right)$. Then there exist $J_{i}$-invariant distributions $E_{i}^{1}, \cdots, E_{i}^{k}$ on $M_{i}$ such that

(2.7.1) the tangent bundle $T M_{i}=E_{i}^{1} \oplus \cdots \oplus E_{i}^{k}$ for sufficiently large $i$.

(2.7.2) the limits of $E_{i}^{j}, 1 \leq j \leq k$, are parallel $J_{\infty}$-invariant distributions of the $C^{1, \alpha}-$ Kähler manifold $\left(X, J_{\infty}, g_{\infty}\right)$.

Proof. Following Proposition 2.3 let $\hat{\xi}^{1}, \cdots, \hat{\xi}^{k} \in H^{1,1}(X)$ denote the limits of $\hat{\xi}_{i}^{1}, \cdots, \hat{\xi}_{i}^{k} \in H^{1,1}\left(M_{i}\right)$. For the sake of simplicity we assume $\xi_{i}^{1}=\omega_{i}$ be the Kähler form of $M_{i}$, and $\hat{\xi}^{1}=\hat{\omega}$ the Kähler form of $X$.

Let $S_{i, 2}, \cdots, S_{i, k}$ be the endomorphisms in (2.6) associated to $\xi_{i}^{2}, \cdots, \xi_{i}^{k}$ with respect to the Kähler form $\omega_{i}$. Since $\hat{\omega}, \hat{\xi}^{2}, \cdots, \hat{\xi}^{k}$ are all parallel, all the eigenvalue functions of $S_{i, j}, j=2, \cdots, k$, converge to constants. Indeed, they converge to the eigenvalue functions of $\hat{S}_{j}, j=2, \cdots, k$, the endomorphisms associated to $\hat{\xi}^{2}, \cdots, \hat{\xi}^{k}$ with respect to $\hat{\omega}$.

Note that every $\hat{S}_{j}, j=2, \cdots, k$, must have at least two different eigenvalues. Otherwise, $\hat{\xi}^{j}$ must be a multiple of $\hat{\omega}$. This is impossible by Proposition 2.3. Therefore $\hat{S}_{j}$ gives a decomposition of $T X$ into at least two parallel eigen distributions. Therefore, if $k \geq 2$ the eigen distributions of $\hat{S}_{2}$ gives a parallel $J_{\infty}$-invariant decomposition $T X=\tilde{E}^{1} \oplus \cdots \oplus \tilde{E}^{r}$ with $r \geq 2$. If $r<k$, the restrictions of $\hat{\xi}^{2}, \hat{\xi}^{3}, \cdots, \hat{\xi}^{k}$ on some factor, $\hat{E}^{s}$, must have rank $>1$. By the above $\hat{E}^{s}$ can be further splitted into parallel eigen distributions. This implies $r \geq k$. Correspondingly, $S_{i, j}$ gives an eigen decomposition $T M_{i}=\tilde{E}_{i}^{1} \oplus \cdots \oplus \tilde{E}_{i}^{r}$ for $i$ sufficiently large so that $\tilde{E}_{i}^{j}$ converges to $\tilde{E}^{j}$. Note that all the distributions are $J_{i}$-invariant.

It suffices to prove $r=k$.

Note that the parallel distributions $\tilde{E}^{1}, \cdots, \tilde{E}^{r}$ are integrable. By Theorem 1.4 $X=X_{1} \times \cdots \times X_{r}$ accordingly so that $\tilde{E}^{j}, j=1 \cdots, r$, are the distributions given by the product foliations. Observe that $h^{1,1}\left(X_{j}\right) \geq 1$ since $X_{j}$ is a compact $C^{1, \alpha}$-Kähler manifold.

Let $\omega_{i, j}$ denote the Kähler forms of the distributions $\tilde{E}_{i}^{j} \subset T M_{i}, j=1 \cdots, r$. Note that $\omega_{i, j}$ is not necessarily a closed form. Clearly, $\omega_{i, j}$ converges to $\hat{\omega}_{j}$, the closed Kähler form of $X_{j}$, for each $j=1, \cdots, k$. Let $\bar{\omega}_{i, j}$ denote the harmonic component of $\omega_{i, j}$ in $H^{1,1}\left(M_{i}\right)$. Obviously, $\bar{\omega}_{i, j}$ also converges to $\hat{\omega}_{j}$. If $r>k$, then $h^{1,1}(X)>k$ and so the vectors $\bar{\omega}_{i, j}, j=1, \cdots, r$, has rank greater than $k$ for $i$ large by Proposition 2.3. This is impossible since $h^{1,1}\left(M_{i}\right)=k$ for all $i$.

Let $M \in \mathcal{M}(n, \Lambda)$ be a Kähler manifold. Assume $h^{1,1}(M)=k$. By the above there is a constant $\varepsilon(n, \Lambda)$ such that, if $H \cdot \operatorname{diam}^{2}(M) \geq \varepsilon(n, \Lambda)$, then Lemma 2.7 implies 
$k$ distributions $E^{1}, \cdots, E^{k}$. Let $\omega_{1}, \cdots, \omega_{k}$ be the Kähler forms of the distributions and let $\bar{\omega}_{1}, \cdots, \bar{\omega}_{k} \in H^{1,1}(M)$ be their $(1,1)$-harmonic components. From the proof of Lemma 2.7 we know that $\bar{\omega}_{1}, \cdots, \bar{\omega}_{k}$ are linear independent. Therefore $\bar{\omega}_{1}, \cdots, \bar{\omega}_{k} \in$ $H^{1,1}(M)$ is a basis.

Let $\phi$ be the Ricci form of $M$. We may write $\phi=a_{1} \bar{\omega}_{1}+\cdots+a_{k} \bar{\omega}_{k}+d \theta$, where $d \theta$ is an exact form.

LEMma 2.8. Let $M \in \mathcal{M}(n, \Lambda)$ be a Kähler manifold with finite fundamental group. Suppose $h^{1,1}(M)=k$. Then there is a positive constant $\varepsilon(n, \Lambda)$ such that if $H \cdot \operatorname{diam}^{2}(M) \geq-\varepsilon(n, \Lambda)$, then $a_{i}>c(n, \Lambda)$ for $i=1, \cdots, k$, where $c(n, \Lambda)$ is a positive constant depending only on $n, \Lambda$.

Proof. We first prove that

$$
\int_{M} \phi \wedge \omega_{1}^{n_{1}} \wedge \cdots \wedge \omega_{i}^{n_{i}-1} \wedge \cdots \wedge \omega_{k}^{n_{k}}>c(n, \Lambda)
$$

for some positive constant as above.

Suppose not. For simplicity we assume a sequence of Kähler manifolds $M_{i}$ with Ricci forms $\phi_{i}$ converging to a $C^{1, \alpha}$-Kähler manifold $X$ such that:

(2.8.1) $H_{i} \geq-1 / i$, the sectional curvature $K_{M_{i}} \leq \Lambda$, and diameter $\operatorname{diam}\left(M_{i}\right) \leq D$;

(2.8.2) $h^{1,1}\left(M_{i}\right)=k$ and $\pi_{1}\left(M_{i}\right)$ are all finite;

(2.8.3) A sequence of $J_{i}$-invaraint distributions $E_{i}^{1}, \cdots, E_{i}^{k}$ in $T M_{i}$ with limit $E^{1}, \cdots, E^{k}$ parallel $J_{\infty}$-distributions of dimensions $n_{1}, \cdots, n_{k}$ respectively.

(2.8.4) The lower limit $\underline{\varliminf}_{i} \int_{M_{i}} \phi_{i} \wedge \omega_{i, 1}^{n_{1}-1} \wedge \cdots \wedge \omega_{i, k}^{n_{k}} \leq 0$, where $\omega_{i, 1}, \cdots, \omega_{i, k}$ are the Kähler forms of the distributions $E_{i}^{1}, \cdots, E_{i}^{k}$.

Let $V\left(M_{i}\right)=\int_{M_{i}} \omega_{i, 1}^{n_{1}} \wedge \cdots \wedge \omega_{i, k}^{n_{k}}$. By Theorem 1.7 the injectivity radius of $M$ has a uniform positive lower bound. Clearly, $V\left(M_{i}\right)$ converges to $V(X)$, the volume of $X$, since $\omega_{i, 1}^{n_{1}} \wedge \cdots \wedge \omega_{i, k}^{n_{k}}$ converges in $C^{1, \alpha}$ (resp. $L^{2, p}$ ) class to the volume form of $X$. Moreover, $\int_{M_{i}} \phi_{i} \wedge \omega_{i, 1}^{n_{1}-1} \wedge \omega_{i, 2}^{n_{2}} \wedge \cdots \wedge \omega_{i, k}^{n_{k}}$ converges to $\lim _{i} a_{i, 1} V(X)$. Therefore the desired result follows from (2.8.0).

Consider the restriction of the Ricci transformation Ric : $T M_{i} \rightarrow T M_{i}$ on the distribution $E_{i}^{1}$. Let $\lambda_{i}^{j}, 1 \leq j \leq n_{1}$, denote the eigenvalues of this restriction. Let $\lambda_{i}^{j,-}=\chi \lambda_{i}^{j}$ where $\chi$ is the character function of the set $\left\{x \in M_{i}: \lambda_{i}^{j}(x)<0\right\}$ (i.e. $\chi$ has value 1 for $x$ in the set and zero otherwise). Since $E_{i}^{j}$ converges in $L^{2, p}$-class to a totally geodesic foliation in $X$ for any integer $p>0$, by (2.8.1) it follows that

$$
\int_{M_{i}}\left|\lambda_{i}^{j,-}\right|^{p} \leq b_{i}(p, n, \Lambda)
$$

where $\left\{b_{i}(p, n, \Lambda)\right\}$ is a sequence of positive constants depending only on $p, n$ and $\Lambda$ which converges to zero when $i$ goes to infinity.

Let $A_{i}=\int_{M_{i}} \phi_{i} \wedge \omega_{i, 1}^{n_{1}-1} \wedge \cdots \wedge \omega_{i, k}^{n_{k}}$. Note that

$$
A_{i}=\frac{1}{\left(n_{1}-1\right) !} \int_{M_{i}}\left(\lambda_{i}^{1}+\cdots+\lambda_{i}^{n_{1}}\right) \omega_{i, 1}^{n_{1}} \wedge \cdots \wedge \omega_{i, k}^{n_{k}}
$$

By (2.8.5) it is clear that $\underline{\lim }_{i} A_{i} \geq 0$. If $\underline{\lim }_{i} A_{i}=0$, passing to a subsequence if necessary we may assume that $\lim _{i} A_{i}=0$.

Let $\lambda_{i}(x)=\max \left\{\lambda_{i}^{1}(x), \cdots, \lambda_{i}^{n_{1}}(x)\right\}$ for $x \in M_{i}$. Using Hölder inequality, (2.8.5) 
and (2.8.6) imply that

$$
\int_{M_{i}}\left|\lambda_{i}\right|^{p} \leq c_{1}(p, n, \Lambda) A_{i}+c_{2}(p, n, \Lambda) b_{i}(p, n, \Lambda)
$$

for all large $i$, where $c_{1}(p, n, \Lambda)$ (resp. $\left.c_{2}(p, n, \Lambda)\right)$ is a positive constant depending only on $p, n$ and $\Lambda$. Therefore $\lambda_{i}$ converges in $L^{p}$-class to zero for any integer $p>0$. This implies that the limit metric $g=g_{\infty}$ on $X_{1}$ (the limit of the distribution $E_{i}^{1}$ ) gives an $L^{2, p}$-weak solution to the Ricci flat equation (2.0)

$$
-\frac{1}{2} g^{i j} \frac{\partial^{2} g_{r s}}{\partial x_{i} \partial x_{j}}+Q\left(\frac{\partial g_{k l}}{\partial x_{m}}\right)=0
$$

By the elliptic regularity we know that the restriction of $g_{i j}$ on $X_{1}$ is a smooth metric with flat Ricci curvature (cf. [An] and Theorem 1.6). Note that the bisectional curvature of $\left(X_{1}, g\right)$ is non-negative. Therefore $\left(X_{1}, g\right)$ has zero bisectional curvature and so $\left(X_{1}, g\right)$ is flat. By the well-known Bieberbach theorem a finite covering space of $X_{1}$ is a torus. In particular, $\pi_{1}\left(X_{1}\right)$ is infinite. Thus $\pi_{1}(X)=\pi_{1}\left(X_{1}\right) \times \cdots \times \pi_{1}\left(X_{k}\right)$ is also infinite. By Theorems 1.3 and 1.7 it follows that $\pi_{1}\left(M_{i}\right) \cong \pi_{1}(X)$ is infinite for $i$ large. A contradiction. The desired result follows.

Lemma 2.9. Let $M \in \mathcal{M}(n, \Lambda)$ be as in Lemma 2.8. Let $\mu(x)=\min \left\{\lambda_{1}(x), \cdots, \lambda_{h}(x)\right\}$ for $x \in M$, where $\lambda_{1}, \cdots, \lambda_{n}$ are the eigenvalue functions of the Ricci transformation Ric $: T M \rightarrow T M$. Then

$$
\int_{M} \mu>c_{0}(n, \Lambda)
$$

a positive constant depending only on $n$ and $\Lambda$.

Proof. Suppose not, there is a sequence of manifolds $M_{i} \in \mathcal{M}(n, \Lambda)$ satisfying (2.8.1) and (2.8.2). Write the Ricci form $\phi_{i}=a_{1} \bar{\omega}_{i, 1}+\cdots+a_{k} \bar{\omega}_{i, k}+d \theta_{i}$, where $d \theta_{i}$ is an exact form. By Lemma $2.8 a_{j}>c>0, j=1, \cdots, k$. Therefore

$$
\lim _{i} \int_{M_{i}} \phi_{i}^{n} \geq c^{n} \alpha(n) \mathrm{V}(X)>0
$$

where $\alpha(n)$ is a positive function depending only on $n$.

Let $\lambda_{i, 1}, \cdots, \lambda_{i, n}$ be the eigenvalue functions of Ric : $T M_{i} \rightarrow T M_{i}$. Note that

$$
\lim _{i} \int_{M_{i}} \lambda_{i, 1} \cdots \lambda_{i, n} \geq c^{n} \beta(n) \mathrm{V}(X)>0
$$

where $\beta(n)$ is a positive function depending only on $n$.

Let $M_{i}^{\geq 0}=\left\{x \in M_{i}: \mu_{i}(x) \geq 0\right\}$ and $M_{i}^{<0}$ be the complement of $M_{i}^{\geq 0}$ in $M_{i}$. Since the sectional curvature $K \leq \Lambda$, it holds that

$$
\begin{gathered}
\left|\int_{M_{i}^{<0}} \lambda_{i, 1} \cdots \lambda_{i, n}\right| \leq \frac{(n-1)}{i}(n \Lambda)^{n-1} V\left(M_{i}\right) \\
\int_{M_{i}^{\geq 0}} \lambda_{i, 1} \cdots \lambda_{i, n} \leq(n \Lambda)^{n-1} \int_{M_{i}^{\geq 0}} \mu_{i}
\end{gathered}
$$


Together with (2.9.1) we get that

$$
\begin{gathered}
\lim _{i} \int_{M_{i}^{\geq 0}} \lambda_{i, 1} \cdots \lambda_{i, n}=\lim _{i} \int_{M_{i}} \lambda_{i, 1} \cdots \lambda_{i, n}-\lim _{i} \int_{M_{i}^{<0}} \lambda_{i, 1} \cdots \lambda_{i, n} \\
\geq c^{n} \beta(n) \mathrm{V}(X)-\lim _{i} \frac{(n-1)}{i}(n \Lambda)^{n-1} \mathrm{~V}\left(M_{i}\right)
\end{gathered}
$$

Now (2.9.3) and (2.9.4) together implies that

$$
\int_{M_{i}} \mu_{i}=\int_{M_{i}^{\geq 0}} \mu_{i}+\int_{M_{i}^{<0}} \mu_{i} \geq \int_{M_{i}^{\geq 0}} \mu_{i}-\frac{n-1}{i} V\left(M_{i}\right)>c_{0}(n, \Lambda)>0
$$

for $i$ sufficiently large. A contradiction. This proves the desired result.

Now we are ready to prove the Theorem 2.5.

Proof of Theorem 2.5. Let $\varepsilon(n, \Lambda)$ be the constant in Lemma 2.9. Let $M \in$ $\mathcal{M}(n, \Lambda)$ be a Kähler manifold such that $H \cdot \operatorname{diam}^{2}(M) \geq-\varepsilon(n, \Lambda)$. Let $\left\{e_{1}, J e_{1}, \cdots, e_{n}, J e_{n}\right\}$ be an orthonormal basis of the tangent space $M_{x}$ with the following property: if $V_{i}=\frac{1}{\sqrt{2}}\left(e_{i}-\sqrt{-1} J e_{i}\right)$, then $\left(\sum_{k} R_{V_{k} \bar{V}_{k}}\right) V_{i}=\lambda_{i} V_{i}$ for all $i$ with $\lambda_{i} \in \mathbb{R}$. Let $\left\{\theta^{i}\right\}$ be dual of $\left\{V_{i}\right\}$ and of type $(1,0)$. Let $\xi$ be a harmonic form of type $(p, 0)$. We may write $\xi=\sum_{I} \xi_{I} \theta^{I}$, where $I$ runs through all strictly increasing multi-indices $\left(i_{1}, \cdots, i_{p}\right)$ and $\theta^{I}=\theta^{i_{1}} \wedge \cdots \wedge \theta^{i_{p}}$. By the Weitzenböck formula we get (c.f. [Wu2] page 313)

$$
-\Delta|\xi|^{2}=\sum_{i}\left|D_{\bar{V}_{i}} \xi\right|^{2}+\sum_{i}\left|D_{V_{i}} \xi\right|^{2}+\sum_{I}\left(\sum_{i \in I} \operatorname{Ric}\left(e_{i}, e_{i}\right)\right)\left|\xi_{I}\right|^{2}
$$

Normalize $\xi$ so that its $L^{2}$-norm is 1 . Integrating (2.10) on $M$ we get that

$$
\int_{M} \mu=0
$$

where $\mu$ is as in Lemma 2.9. A contradiction to Lemma 2.9. The desired result follows.

\section{Proofs of Theorems A, B, C, D.}

Proof of Theorem $A$. Let $k=h^{1,1}(M)>1$. Assume not, then we get a sequence of simply connected Kähler manifolds $M_{i}$, such that

(3.1) $H_{i} \cdot \operatorname{diam}^{2}\left(M_{i}\right) \geq-(0.1)^{i}$ and $K_{i} \leq \Lambda$;

(3.2) $h^{1,1}\left(M_{i}\right)=k$;

(3.3) $M_{i}$ does not diffeomorphic to the product of $k$ simply connected $C^{1, \alpha}$-Kähler manifolds with second Betti number 1 for any $i$.

By Theorems 1.3, 1.5 and 1.7 the limit $X$ of the sequence $M_{i}$ is a $C^{1, \alpha}$ Kähler manifold of dimension $n$. It suffices to prove that $X$ is diffeomorphic to a product $X_{1} \times \cdots \times X_{k}$, where each factor $X_{i}$ is a $C^{1, \alpha}$-Kähler manifold with $b_{2}\left(X_{i}\right)=1$.

By Lemma 2.7 we get $k$ parallel distributions of $X$, which implies that the $C^{1, \alpha}$ Kähler manifold $X$ is isometric to the product $X_{1} \times \cdots \times X_{k}$ by Theorem 1.4. We now verify that $b_{2}\left(X_{1}\right)=\cdots=b_{2}\left(X_{k}\right)=1$, which clearly implies the desired result.

By Theorem 2.5 we know that $b_{2}\left(M_{i}\right)=h^{2,0}\left(M_{i}\right)+h^{1,1}\left(M_{i}\right)+h^{0,2}\left(M_{i}\right)=$ $h^{1,1}\left(M_{i}\right)=k$ using Hodge duality. Clearly $b_{2}\left(X_{j}\right) \geq h^{1,1}\left(X_{j}\right) \geq 1$ since $X_{j}$ is a $C^{1, \alpha}$-Kähler manifold. Therefore $b_{2}(X)=b_{2}\left(X_{1}\right)+\cdots+b_{2}\left(X_{k}\right) \geq k$ and the equality 
holds only if $b_{2}\left(X_{j}\right)=1$ for $j=1, \cdots, k$. Since $b_{2}\left(M_{i}\right)=b_{2}(X)$ for $i$ sufficiently large, the desired result follows.

Proof of Theorem B. By the Anderson's regularity theorem we know that $g_{\infty}$ is a smooth Kähler metric on $X$. Applying Mok [Mo] the desired result follows.

Proof of Theorem $C$. We claim that there is a constant $\varepsilon(n, \Lambda)$ such that if $M \in$ $\mathcal{M}(n, \Lambda)$ with $H \cdot \operatorname{diam}^{2}(M) \geq-\varepsilon(n, \Lambda)$, then there are $k=h^{1,0}$ pointwisely linearly independent holomorphic 1-forms $\theta_{1}, \cdots, \theta_{k}$. The proof of this fact is exactly the same as [Ya] for harmonic 1-forms. For such holomorphic 1-forms $\theta_{1}, \cdots, \theta_{k}$, the Albanese map $\pi: M \rightarrow \mathcal{J}(M)$ is a holomorphic submersion. Therefore it is a holomorphic bundle since $M$ is compact. This completes the proof.

Proof of Theorem D. Suppose not. For simplicity we may assume a sequence of compact Kähler manifolds $M_{i}$ such that $H_{i} \geq-\frac{1}{i}$ and $\operatorname{diam}\left(M_{i}\right) \leq 1, K_{i} \leq \Lambda$ but $h^{1,1}\left(M_{i}\right)>n$. Let $\xi_{i, 1} \cdots, \xi_{i, l}$ be $l$ (where $l>n$ ) linearly independent real harmonic $(1,1)$-forms such that the normalized $L^{2}$-norms, $\left\|\xi_{i, 1}\right\|_{0,2} / \sqrt{\operatorname{vol}\left(M_{i}\right)}$, are all equal to 1.

Consider harmonic coordinate covers of $M_{i}$ with radii $r$ and uniform number $k$, $B_{i}^{1}(r), \cdots, B_{i}^{k}(r)$. The injectivity radii of points in the radii $r$ balls with respect to the lifted metrics on $B_{i}^{1}(2 r), \cdots, B_{i}^{k}(2 r)$ have a uniform positive lower bound. Therefore the lifting of the harmonic $(1,1)$-forms $\xi_{i, j}$ on $B_{i}^{1}(r), \cdots, B_{i}^{k}(r)$ converge to $l$ linearly independent parallel $(1,1)$-forms by Lemma 2.4. By Lemma 2.7 if $i$ is sufficiently large $T M_{i}$ may be decomposed into the direct sum of $l J_{i}$-invariant distributions. For dimension reasoning this clearly implies $l \leq n$. A contradiction.

\section{REFERENCES}

[ADN] S. Agmon; A. Douglis; L. Nirenberg, Estimates near the boundary for solutions of elliptic partial differential equations satisfying general boundary conditions II, Commun. on Pure. Appl. Math., 17 (1964), pp. 35-92.

[An] M. ANDERSON, Convergence and rigidity of manifolds under Ricci curvature bounds, Invent. Math., 102 (1990), pp. 429-445.

[BG] C. BEnson; C. Gordon, Kähler and symplectic structures on nilmanifolds, Topology, 27 (1988), pp. 513-518.

[Be] A. L. BEsse, Einstein Manifolds, Springer-Verlag (1987).

[Ch] J. Cheeger, Finiteness theorems for Riemannian manifolds, Amer. J. Math., 92 (1970), pp. $61-75$.

[CC] J. Chenger; T. Colding, Almost rigidity of wraped product and the structure of spaces with Ricci curvature bounded below, C. R. Acad. Paris, t 320 (1995), pp. 353-357.

[CG] J. CheEger; D. GROMOLL, On the structure of complete manifolds of nonnegative curvature, Ann. of Math., 96 (1972), pp. 413-443.

[FR] F. FANG; X. Rong, Fixed point free circle actions and finiteness theorems, Comm. Contemp. Math., 2 (2000), pp. 75-86.

[FY] K. FUKAYA; T. YAMAGUCHI, The fundamental groups of almost non-negatively curved manifolds, Ann. of Math., 136 (1992), pp. 253-333.

[Ga] S. GALlot, A Sobolev inequality and some geometric applications, Spectra of Riemannian manifolds, Kaigai, Tokyo (1983), pp. 45-55.

[GK] S. Goldberg; S. Kobayashi, Holomorphic bisectional curvature, Jour. Diff. Geom., 1 (1967), pp. 225-233.

[GLP] M. GRomov, J. LAFONTAINE; P.PANSU, Structures metriques pour les varietes riemannienes, CedicFernand Paris (1981). 
[Ha] K. Hasegawa, Minimal models of nilmanifolds, Proc. Amer. Math. Soc., 106 (1989), pp. 6571.

[HSW] A. HOWARD, B. SMYTH, H. Wu, On compact Kähler mnaifolds of non-negative bisectional curvature, Acta. Math. (1981), pp. 51-56.

[Jo] J. Jost, Harmonic mappings between Riemannian manifolds,, Proc. Centre for Math. Analysis, Australian Nat. Univ., 4 (1983).

[JK] J. JOST; H. KARCHER, Geometrische methoden zur Gewinnug von a-priori- Schrander für harmonitsche Abbildungen, Manuscripta. Math., 40 (1982), pp. 27-77.

[Li] P. LI, On the Sobolev constant and the p-spectrum of a compact Riemannian manifold, Ann. Sci. École Norm. Sup., 13 (1980), pp. 451-468.

[Lic] P. Lichnerowicz, Théorie globale des connexions et des groupes d'holonomie, Dunod, Paris (1955).

[Mok] N. MoK, The uniformization theorem for compact Kähler manifolds of non-negative holomorphic bisectional curvature, Jour. Diff. Geom., 27 (1988), pp. 179-214.

[Mo] S. MorI, Projective manifolds with ample tangent bundles, Ann. of Math. (2), 110 (1979), pp. 593-606.

[NW] A. NiJenhuis, W.B. Woolf, Some integration problems in almost complex manifolds, Ann. of Math. (2), 77 (1963), pp. 424-483.

[Pe] P. Petersen, Riemannian geometry, GTM, Springer-Verlag (1997).

[SY] Y. -T. SIU, S. -T. YAU,, Compact Kähler manifolds with positive bisectional curvature, Invent. Math., 59 (1980), pp. 189-204.

[Wu 1] H. Wu, On compact Kähler manifolds of non-negative bisectional curvature II, Acta Math. (1981), pp. 57-70.

[Wu 2] H. Wu, The Bochner technique in Differential Geometry, Mathematical Reports, Harwood Academic Publishers, 3, Part 2 (1988), pp. 289-538.

[Ya] T. YAMAGUChI, Manifolds of almost nonnegative Ricci curvature, Jour. Diff. Geom., 28 (1988), pp. 157-167. 